\title{
Atividade extensionista em serviços de saúde em um município do Rio Grande do Sul
}

\author{
Rosângela Marion da Silva \\ cucasma@terra.com.br
}

Helena Carolina Noal

helenacnoal@gmail.com

Maiara Leal da Trindade

maiaralealt7@gmail.com

Universidade Federal de Santa Maria | Brasil
Francine Ziegler Leal
francineziegler@bol.com.br

Juliana Ebling Brondani

jubrondani@yahoo.com.br

\section{Clarissa Potter}

cissa.pr.enf@gmail.com

\section{Resumo}

Os trabalhadores de saúde são atores fundamentais no estabelecimento de um vínculo com o usuário e na responsabilização pela continuidade da assistência por meio da orientação quanto ao percurso na rede a fim promover a integralidade do cuidado. Objetiva-se relatar a experiência da elaboração de materiais informativos para trabalhadores de saúde de um município do Rio Grande do Sul. Ocorreu entre julho e agosto de 2014 e entre abril e dezembro de 2015 por um grupo de especializadas de um programa de residência, e envolveu mais de 32 trabalhadores de saúde distribuídos em 16 unidades de saúde. Os resultados foram à produção de dispositivos informativos e discussões sobre o tema.

\section{Palavras-chave}

Assistência à saúde; Educação continuada; Assistência integral à saúde. 


\section{Introdução}

As Redes de Atenção à Saúde (RAS) surgiram como uma nova forma de organizar e integralizar o sistema de atenção à saúde, o que torna possível responder com efetividade, eficiência, segurança, qualidade e equidade às necessidades de saúde da população brasileira (MENDES, 2011). As RAS, coordenadas pela Atenção Primária à Saúde (APS), permitem ofertar atenção contínua e integral para a população, cujas responsabilidades geram valor para os usuários (BRASIL, 2010; MENDES, 2011).

Para que essa ideia se efetive é imprescindível à ação dos trabalhadores de saúde, atores fundamentais no estabelecimento de um vínculo com o usuário e na responsabilização pela continuidade da assistência por meio da orientação quanto ao percurso na rede, a fim de contemplar as suas necessidades. Para que isso aconteça, é essencial organizar os processos de trabalho, pois é por meio deles que se produz o cuidado aos usuários (MALTA; MERHRY, 2010; FRANCO; FRANCO, 2014).

Pesquisa realizada com usuários hospitalizados sobre o atendimento recebido nos serviços de saúde identificou práticas que desfavorecem o estabelecimento e a articulação das redes de atenção à saúde, como a comunicação deficiente ou inexistente por parte dos trabalhadores dos serviços e a falta de humanização na assistência, o que dificulta o seu caminhar na rede e incentiva a busca por atendimento em instituições hospitalares (SIQUEIRA et al., 2016). É neste sentido que a atenção básica e os diversos níveis de especialidades e de apoio diagnóstico necessitam operar mudanças frente à conformação dos serviços, criação e fortalecimento de novas práticas em saúde.

Acredita-se que esta conduta atente para a superação de um atendimento fragmentado e impessoal. Soma-se a isso a necessidade de integralidade da atenção à saúde, que deve ser vista sob o aspecto não apenas da organização dos recursos disponíveis, mas, especialmente, do fluxo do usuário dentro da RAS (FRANCO; JÚNIOR, 2014). Esse fluxo será efetivo se os trabalhadores da saúde conhecerem a articulação entre a rede e os serviços por ela oferecidos.

Ferreira, Varga e Silva (2009) destacam o trabalho desenvolvido por uma equipe multiprofissional deve buscar a singularidade e integralidade da assistência, sendo um meio importante para a reorganização dos processos de trabalho. A composição de uma equipe multiprofissional, complementam, favorece a troca de informações e possibilita a 
Atividade extensionista em serviços de saúde em um município do Rio Grande do Sul

elaboração de um plano terapêutico, reforçando a cooperação como parte estratégica do fazer em grupo. Para Silva e Ramos (2011, p. 253), a perspectiva da integralidade requer um refletir, "um repensar dos profissionais na busca por caminhos e estratégias que possam modificar o atual cenário e preencher as lacunas existentes no cuidado prestado e na articulação entre seus diferentes níveis", possibilitando a promoção da saúde e, consequentemente, a redução de reinternações.

Assim, uma das formas para capacitar os trabalhadores dos serviços para o encaminhamento dos usuários é utilizar como estratégia dispositivos informativos oferecidos pela rede para que os auxiliem no encaminhamento dos usuários com vistas a contribuir para a continuidade do cuidado. Uma das formas para se efetivar essa ideia é a realização de ações extensionistas, pois ela estimula a interação dialógica entre a universidade e a sociedade. Com o intuito de promover a troca de saberes, priorizando ações que minimizem as desigualdades, buscando atingir a integralidade da assistência em saúde com atuação multiprofissional e interdisciplinar.

Neste contexto, almeja-se que a prática extensionista seja reflexo de ações de impacto social, e que promova discussões acerca da realidade, convergindo por meio da tríade ensino-pesquisa-extensão. Com isso, deseja-se obter o reconhecimento da universidade como um órgão legítimo de construção e que, por meio da educação, possa-se atender as demandas gerais da população promovendo mudanças no contexto social.

\section{Objetivo}

Relatar a experiência da elaboração de materiais informativos para trabalhadores de saúde de um município do Rio Grande do Sul.

\section{Método}

Este relato está vinculado ao projeto de extensão Atividades Educativas em Saúde na Perspectiva Multiprofissional, registrado no Gabinete de Projetos de uma instituição federal sob o número 039292, e que foi contemplado com financiamento do Fundo de Incentivo à Extensão (FIEX) durante o ano de 2015. De acordo com a Política de 
Atividade extensionista em serviços de saúde em um município do Rio Grande do Sul

Extensão da instituição, a atuação da extensão constitui-se, dentre outras ações, na criação de um produto acadêmico para difusão e divulgação de informações. A ação está classificada na área temática de extensão saúde, linha de extensão desenvolvimento de produtos e saúde humana e é caracterizada como publicação e divulgação de produtos acadêmicos (UFSM, 2007). 0 produto acadêmico produzido é um manual do tipo cartilha e foi direcionada para os trabalhadores de saúde da RAS de um município do interior do Rio Grande do Sul com a finalidade de capacitá-los e atualizá-los para o encaminhamento do usuário na RAS, visando à integralidade do cuidado.

0 município em questão abrange oito regiões administrativas (região oeste, centro-oeste, norte, leste, centro, nordeste, sul, centro-leste) e uma zona rural. 0 serviço de saúde conta com Unidades Básicas de Saúde (UBS) e unidades da Estratégia de Saúde da Família (ESF), distribuindo-se entre as oito regiões supracitadas.

A prática intervencionista foi realizada entre julho e agosto de 2014 e abril a dezembro de 2015, junto às unidades de saúde e um hospital universitário, contemplando 16 serviços. Os locais escolhidos atenderam ao critério de serem unidades de referência aos usuários do município que estiveram hospitalizados com alterações vasculares no período de julho a agosto do ano de 2014. Para Malta e Merhy (2010) o hospital, representante da atenção terciária, possui papel fundamental para a articulação com a RAS, especialmente no momento da alta hospitalar do usuário. Sendo primordial estabelecer a contra referência para a rede básica à qual o usuário já está vinculado, para possibilitar o processo de continuidade do cuidado.

A gênese desta atividade de extensão aconteceu durante a vivência das participantes deste relato como especializadas de um programa de residência que realizaram suas atividades em unidades de saúde e em uma instituição hospitalar. Foi no momento em que perceberam o não encaminhamento dos usuários às unidades de referência e a serviços de saúde da RAS, bem como ouviram relatos de trabalhadores sobre os motivos para o não encaminhamento: desconhecimento dos serviços da RAS.

Sobre isso, pesquisadores referenciam que a instituição hospitalar é um local que deveria trabalhar de forma integrada com a atenção primária em saúde para atender às necessidades dos usuários que a procuram, mas o que se percebe é um sistema sem comunicação e desarticulado dos demais serviços de atenção à saúde (DUARTE, NORO; 2013). 
Atividade extensionista em serviços de saúde em um município do Rio Grande do Sul

A partir disso, a equipe multiprofissional envolvida com a proposta promoveu a construção de dispositivos informativos dos serviços ofertados pelo Sistema Único de Saúde (SUS) do município com o intuito de auxiliar e instrumentalizar o trabalhador da saúde no encaminhamento dos usuários. Os trabalhadores que participaram da ação de extensão foram enfermeiros, técnicos e auxiliares de enfermagem, assistentes sociais e médicos.

\section{Implementação da proposta}

Os dispositivos informativos constituíram-se de uma cartilha com os principais serviços de saúde na RAS do município, com ênfase no atendimento ao adulto e idoso, pessoas geralmente com maior acometimento de alterações vasculares, e um banner informativo.

Para a confecção da cartilha Serviços da Rede de Atenção à Saúde, buscaram-se informações sobre os serviços de saúde da RAS do município no site da Prefeitura Municipal, na Secretaria de Desenvolvimento Social e no Plano Municipal de Saúde 2012-2016. Nesta cartilha, direcionada aos profissionais de saúde, constavam o número de telefone, endereço dos serviços de saúde do município e a finalidade do serviço em questão. 0 material foi subdividido em áreas profissionais a fim de melhor organizar os dados, como, por exemplo: Assistência Social (Centro de Referência Especializado de Assistência Social - CREAS, entre outros); Idosos (Delegacia de Proteção ao Idoso, entre outros); Saúde Mental (Alcoólicos Anônimos, Conselho Municipal de Entorpecentes, Centro de Atenção Psicossocial, entre outros); Associação De Deficientes Físicos; Associação dos Surdos e unidades de saúde do município. Também se optou por elaborar o banner, que continha um resumo dos serviços existentes na cartilha. 0 banner foi construído para facilitar a visualização das informações pelos trabalhadores de saúde.

A produção do material foi realizada pelos autores deste relato, com posterior diagramação por uma acadêmica voluntária de um curso de Desenho Industrial. Foi realizada com o auxílio de recursos do programa de residência. Os materiais produzidos foram entregues as 16 unidades de saúde, o que envolveu diretamente 32 profissionais 
Atividade extensionista em serviços de saúde em um município do Rio Grande do Sul

de saúde capacitados para os encaminhamentos, pois os materiais confeccionados eram entregues para, no mínimo, dois trabalhadores por serviço em encontros pré-agendados.

Para efetivar a ação extensionista, a equipe de trabalho primeiramente buscava conhecer o fluxo de encaminhamento do usuário na RAS pelo trabalhador. Após essa conversa inicial, realizava-se uma breve apresentação da proposta do projeto com enfoque na necessidade de encaminhamento do usuário para atingir a integralidade do cuidado. Esse momento objetivou sensibilizá-lo para a orientação do percurso na RAS. A seguir, apresentavam-se os dispositivos produzidos (cartilha e banner).

Os trabalhadores contatados expressaram, em muitas situações, surpresa frente ao quantitativo de serviços oferecidos e disponibilizados pelo município, relatando que os materiais iriam auxiliá-los no encaminhamento do usuário, não se limitando somente ao atendimento da unidade. Da mesma forma, buscou-se frisar a importância da socialização das informações entre os demais trabalhadores das unidades participantes para auxílio na organização do serviço, sendo o maior beneficiado o usuário.

Menozzi (2013) afirma que para um sistema de referência e contra referência funcionar é necessário muito mais que estabelecimento de fluxos e informações, é preciso a preponderância dos processos educativos das equipes, a difusão de conhecimentos para a melhoria da qualidade do atendimento em saúde e, para isso, a instrumentalização nos processos de saúde torna-se premente. Frente a isso, autores sinalizam que é necessário oferecer aos trabalhadores de saúde condições mínimas de atuação, com o objetivo de efetivar as ações de saúde, o que possibilita o alcance dos objetivos traduzidos pela pactuação da atenção básica, com a finalidade de atender às necessidades de cada localidade (SILVA, MENDES, TORRES; 2012).

Destaca-se que, além dos serviços de saúde, a equipe de trabalho dirigiu-se até o Núcleo de Educação Permanente da Secretaria de Saúde do município, órgão responsável pela organização e elaboração de Planos e Políticas Públicas voltadas para a promoção, prevenção e assistência à saúde, disponibilizando-se a participar das ações de educação permanente com os trabalhadores das unidades de saúde, com o intuito de sensibilizar os trabalhadores para o desenvolvimento do trabalho, promover a articulação entre os profissionais e para divulgar o material produzido. Segundo o Ministério da Saúde (2012), são atribuições da Secretaria Municipal de Saúde organizar o SUS no âmbito municipal e viabilizar o desenvolvimento de ações de saúde por meio 
Atividade extensionista em serviços de saúde em um município do Rio Grande do Sul

de unidades estatais ou privadas, priorizando as entidades filantrópicas.

A elaboração dos dispositivos informativos, que neste caso são a cartilha e o banner, teve como objetivo aproximar os profissionais e sensibilizá-los para os serviços oferecidos na rede visando à integralidade no acesso aos usuários e à qualidade da assistência à saúde. Essa atividade contribuiu para a produção de um projeto de pesquisa guarda-chuva, que culminou na elaboração e apresentação de três Trabalhos de Conclusão de Residência.

\section{CONCLUSÃO}

Esta ação possibilitou aos trabalhadores de saúde de um município informações sobre os serviços oferecidos na RAS, o que, acredita-se, pode contribuir para o fortalecimento do trabalho em redes e na busca pela integralidade da assistência em saúde.

Foi percebida deficiência de comunicação entre as diferentes densidades tecnológicas, e identificou-se a necessidade de educação permanente para atualização dos profissionais quanto aos serviços oferecidos pela rede, importantes aspectos que precisam de investimento por parte dos gestores.

Vale ressaltar que este trabalho apresentou algumas limitações, como a dificuldade encontrada para conseguir contato telefônico com os responsáveis pelas unidades, o deslocamento para chegar às unidades de saúde e o tempo disponível dos profissionais para participar da intervenção. Como pontos positivos, citam-se a boa receptividade, a motivação e o interesse dos profissionais sobre a temática, o que certamente beneficiará os usuários atendidos.

Acredita-se que esta atividade beneficiou três esferas da sociedade: os profissionais, pela disponibilidade de informações que podem auxiliar seu trabalho; acadêmicos de graduação, pois enriqueceu a formação no que se refere ao trabalho interdisciplinar; e os usuários dos serviços de saúde, que poderão ter atendimento mais integral e humanizado.

Para a Enfermagem, estes materiais são uma oportunidade de integralização e continuidade do cuidado aos usuários, melhorando o atendimento nos serviços oferecidos pelo Sistema Único de Saúde. Por fim, sugere-se a continuidade desta 
Atividade extensionista em serviços de saúde em um município do Rio Grande do Sul

proposta com vistas a fortalecer a comunicação entre as diferentes densidades tecnológicas da região e da Rede de Atenção à Saúde. Deve-se considerar a continuidade desta atividade extensionista para ampliar o número de serviços de saúde atendidos; contribuir para o processo de integração ensino-serviço; e potencializar a formação acadêmica, pois, além de ser uma das protagonistas do processo de transformação da realidade do município, fez aproximações do ensino, pesquisa e extensão.

\section{Referências}

BRASIL. Portaria № 4.279, de 30 de dezembro de 2010. Estabelece diretrizes para a organização da Rede de Atenção à Saúde no âmbito do Sistema Único de Saúde (SUS). Acesso em 27 de outubro de 2014. Disponível em: http://conselho.saude.gov.br/ultimas_noticias/2011/img/07_jan_portaria4279_301210 .pdf.

Secretaria de Atenção à Saúde. Departamento de Atenção Básica. Documento de diretrizes para o cuidado das pessoas com doenças crônicas nas Redes de Atenção à Saúde e nas linhas de cuidado prioritárias. Brasília; 2012.

Ministério da Saúde. Portaria № 483, de 1ํ de abril de 2014. Redefine a Rede de Atenção à Saúde das pessoas com Doenças Crônicas Não Transmissíveis no âmbito do Sistema único de Saúde (SUS) e estabelece diretrizes para a organização das suas linhas de cuidado. Brasília; 2014.

DUARTE MLC, NORO A. Humanização do atendimento no setor de radiologia: dificuldades e sugestões dos profissionais de enfermagem. Cogitare Enfermagem, v. 18, n. 3, p. 532-538; 2013.

FERREIRA, R.C.; VARGA, C.R.R., SILVA, R.F. Trabalho em Equipe Multiprofissional: A perspectiva dos residentes médicos em Saúde da Família. Ciência \& Saúde Coletiva, v. 14, p. 1421-28; 2009.

LAVRAS, C. Atenção Primária à Saúde e a Organização de Redes Regionais de Atenção à Saúde no Brasil. Saúde e Sociedade, v. 20, n. 4, p. 867-874; 2011.

MALTA, D. C.; MERHY, E. E. O percurso da linha do cuidado sob a perspectiva das doenças crônicas não transmissíveis. Interface - Comunicação, Saúde, Educação, v.14, n. 34, p. 593-605; 2010.

MENDES, E.V. As redes de atenção à saúde. / Eugênio Vilaça Mendes. Brasília: Organização Pan-Americana da Saúde; 2011. 
MENOZZI, K. A. B. S. o sistema de referência e contra-referência no contexto da equipe multiprofissional de saúde. 2013, 181 p. Dissertação (Mestrado profissional em Enfermagem). Faculdade de Medicina de Botucatu, Botucatu, 2013.

FRANCO, C. M.; FRANCO, T. B. Linhas do cuidado integral: uma proposta de organização da rede de saúde. Acesso em 27 de outubro de 2014. Disponível em: http://www.saude.rs.gov.br/upload/1337000728_Linha\%20cuidado\%20integral\%20c onceito $\% 20$ como $\% 20$ fazer.pdf.

FRANCO, T. B.; JÚNIOR, H.M.M. Linhas do cuidado integral: uma proposta de organização da rede de saúde. Acesso em 28 de outubro de 2014. Disponível em: http://www1.saude.rs.gov.br/dados/1311947118612INTEGRALIDADE\%20NA\%20ASS IST\%CANCIA\%20\%C0\%20SA\%DADE\%20-\%20T\%FAlio.pdf

SILVA, L.M.S.; MENDES, E.P.; TORRES, R.A.M. Trabalho interdisciplinar na estratégia saúde da família: enfoque nas ações de cuidado e gerência. Revista de Enfermagem da UERJ, v 20, n esp. 2, p. 784-788; 2012.

SILVA, R. V. G. O.; RAMOS, F. R. S. Processo de alta hospitalar da criança: percepções de enfermeiros acerca dos limites e das potencialidades de sua prática para a atenção integral. Texto Contexto Enferm, v. 20, n. 2, p. 247-254; 2011.

SIQUEIRA, al. Percepção de usuários hospitalizados sobre o atendimento nos serviços de saúde. Revista de Enfermagem da UFSM, v. 6, n. 4, p. 471 - 481; 2016.

UFSM. Política de Extensão da UFSM; 2007.

WHO.WORLD HEALTH ORGANIZATION. Non communicable diseases country profiles 2014. Acesso em: 27 de outubro de 2014. Disponível em: http://apps.who.int/iris/bitstream/10665/128038/1/9789241507509_eng.pdf?ua=1. 
Extension activity in health services in a city of Rio Grande do Sul

\begin{abstract}
Health workers are key actors in establishing a link with the user and in the responsibility for the continuity of care through guidance on the way in the public health network in order to promote the integrality of care. The objective was to report the experience of the elaboration of informative materials for health workers of a city in Rio Grande do Sul. It occurred between July and August of 2014 and between April and December of 2015 by a group of specialists of a program of residence, and involved more than 32 health workers distributed in 16 health units. The results were the production of informative devices and discussions on the subject.
\end{abstract}

\section{Keywords:}

Delivery of Health Care, Education, Continuing, Comprehensive health care.

Original submetido em: 3 fev. 2016

Aceito para publicação em: 24 maio 2016
Actividad de ampliación de los servicios de salud en la ciudad de Río Grande do Sul

\section{RESUMEN}

Trabajadores de la salud son actores clave en el establecimiento de un enlace con el usuario y la rendición de cuentas para la continuidad de la atención através de la orientación en cuanto a la red de rutas con el fin de promover la atención integral. El objetivo es dar a conocer la experiencia de la preparación de materiales de información para los trabajadores de la salud en una ciudad en Rio Grande do Sul. Se le ocurrió entre julio y agosto de 2014 y entre abril y diciembre de 2015 mediante un grupo de especializandas de un programa de residencia, y participan más de 32 trabajadores de la salud distribuidos en 16 unidades de salud. Los resultados fueron la producción de dispositivos de información y debates sobre el tema.

\section{Palabras clave:}

Prestación de Atención de Salud; Educación Continua, Atención integral de salud.

Sobre as autoras:

\section{Rosângela Marion da Silva}

Enfermeira, doutora em Ciências. Professora adjunta do Departamento de Enfermagem da Universidade Federal de Santa Maria; Grupo de Pesquisa Trabalho, Saúde e Enfermagem.

\section{Helena Carolina Noal}

Enfermeira, mestre em Enfermagem; Hospital Universitário de Santa Maria - Universidade Federal de Santa Maria.

\section{Maiara Leal da Trindade}

Acadêmica de Enfermagem, Universidade Federal de Santa Maria, bolsista de extensão.

\section{Francine Ziegler Leal}

Assistente social, especialista em Gestão e Atenção Hospitalar no

Sistema Único de Saúde; Universidade Federal de Santa Maria. 
Atividade extensionista em serviços de saúde em um município do Rio Grande do Sul

\section{Juliana Ebling Brondani}

Nutricionista, doutoranda em Farmacologia; Universidade Federal de Santa Maria.

\section{Clarissa Potter}

Enfermeira, especialista em Gestão e Atenção Hospitalar no Sistema Único de Saúde; Universidade Federal de Santa Maria. 